\title{
Singular Points of Reactive Distillation Systems
}

\author{
Zhiwen Qi ${ }^{\text {a }}$, Dietrich Flockerzi ${ }^{\text {a }}$ and Kai Sundmacher ${ }^{\text {a, b, * }}$
}

a Max-Planck-Institute for Dynamics of Complex Technical Systems, Sandtorstrasse 1, D-39106 Magdeburg, Germany

b Otto-von-Guericke-University Magdeburg, Process Systems Engineering, Universitätsplatz 2, D-39106 Magdeburg, Germany

\section{Corresponding author:}

Professor Dr.-Ing. Kai Sundmacher

Max-Planck-Institute for Dynamics of Complex Technical Systems,

Sandtorstrasse 1, D-39106 Magdeburg, Germany

Phone: +49-391-6110351, Fax: +49-391-6110353

E-mail: sundmacher@mpi-magdeburg.mpg.de 


\section{Abstract}

For the conceptual design of countercurrently operated reactive distillation columns, fast methods are needed to estimate potential top and bottom products. The possible column bottom product composition can be determined from the stable singular points of a batch reactive reboiler. In a similar manner the top product composition can be obtained from the stable singular points of a batch reactive condenser. Geometrically, the singular points of both batch processes are located on a common potential singular point surface (PSPS) whose trajectory depends on the reaction stoichiometry and the phase equilibria. At the singular points, the PSPS intersects a reaction kinetic surface which is dependent on the reaction rate expression and the phase equilibrium of a reference component. Based on the singularity analysis, a single-feed reactive distillation column can be designed. Several hypothetical and real reaction systems are analyzed to illustrate the singularity analysis and the design procedure.

\section{Topical Heading: Process Systems Engineering}

Keywords: Azeotrope; Reactive Distillation; Reactive Condenser; Reactive Reboiler; Singularity Analysis, Feasible Split

\section{Introduction}

Today there is an increasing interest in the theoretical study and the practical application of reactive distillation processes (Malone and Doherty, 2000; Sharma and Mahajani, 2003). However, very often reactive distillation is not proper for a liquid-phase chemical process since combing reaction and distillation may be not advantageous. Therefore, it is important to quickly estimate whether reactive distillation is a suitable process concept based on minimal information on the physical and chemical properties. As presented by Gadewar et al. (2003), 
an effective way of decomposing the design and development of reactive distillation involves four stages. The first stage deals with the analysis of feasibility, i.e. the analysis of possible top and bottom products. Once reactive distillation is verified to be unsuitable, alternatives should be explored.

Since the simple reactive distillation in a batch vessel can reveal important aspects for a continuously operated column, several groups have worked on this topic. A concept widely used in recent years is Residue Curve Mapping (RCM). The presence of singular points in the RCM has been proved in experiments (Song et al., 1997). For the singular points of a chemical equilibrium controlled reaction system, the terminology reactive azeotrope was introduced (Barbosa and Doherty, 1987, 1988; Ung and Doherty, 1995a). The condition for reactive azeotropy was derived by Ung and Doherty (1995b) in terms of transformed variables, i.e. $X_{i}=Y_{i}$.

So far, most attention was paid to chemical equilibrium controlled systems. However, singular points exist in kinetically controlled systems, too. Rev (1994) used the term kinetic azeotropy for the more general situation of the simultaneous occurrence of a chemical reaction and a separation process. Hauan et al. (1996 and 2000) adopted the term reactive fixed points for their analysis method. Moreover, Venimadhavan et al. (1999) and Qi et al. (2002) studied bifurcations of singular points in kinetically controlled homogeneous and heterogeneous systems, respectively. However, up to now, there is no theory to properly describes and link all kinds of azeotropes, i.e. non-reactive, kinetic and reactive azeotropes.

Since the singularity analysis of batch reactive distillation yields only the possible bottom composition of a continuous reactive distillation column, a generalized method is needed for predicting both, the potential top products as well as the potential bottom products of reactive distillation columns. For this purpose, Chadda et al. (2001) proposed an approach to represent the rectifying section and the stripping section of a column by two isobaric flash cascades. 
The stable nodes of the vapor-fed rectifying cascade and the liquid-fed stripping cascade yield potential distillate and bottom products, respectively. The feasible split can be determined from the flash cascade trajectories in combination with the overall column mass balances.

In this contribution, a new batch process is introduced, namely a reactive condenser, to identify potential top products and a reactive reboiler is used to identify the potential bottom products. The latter process is identical to the one considered in previous works (Venimadhavan et al., 1994; Thiel et al., 1997; Qi et al., 2002). Singular points at nonreactive, at kinetically controlled and also at chemical equilibrium controlled operating conditions can be analyzed. All non-pure-component singular points, except the pole of stoichiometric lines, are regarded as azeotropes in this work. As shown below, all singular points of the batch reactive condenser and the batch reactive reboiler are located on a common surface. However, the exact locations of the singular points on this surface is fixed by two different reaction kinetic surfaces. Based on the stable singular points, feasible splits of a fully reactive distillation column can be predicted.

\section{Model Formulation}

The considered reactive condenser and reactive reboiler are depicted in Figure 1. In both batch processes, the chemical reaction is enhanced by a catalyst with constant holdup $H_{c a t, c}$ in the condenser and constant holdup $H_{c a t, r}$ in the reboiler. The liquid phase itself can be either homogeneous or heterogeneous, i.e. it can split into two liquid sub-phases. In these subphases, the catalyst activity can differ (Qi and Sundmacher, 2002). Moreover, phase equilibrium is established between the liquid phase and the vapor phase, i.e $y_{i}=y_{i}(\mathbf{x})$, and also between the liquid sub-phases arising from a possible phase splitting $x_{i}^{\prime}=x_{i}^{\prime}\left(\mathbf{x}^{\prime \prime}\right)$. The following single reversible chemical reaction is considered in the liquid phases:

$$
v_{1} A_{1}+v_{2} A_{2}+\ldots+v_{N C} A_{N C} \Leftrightarrow 0
$$


where $v_{i}$ is the stoichiometric coefficient of components $i\left(v_{i}<0\right.$ for reactants, $v_{i}>0$ for products). The summation of $v_{i}$ yields $v_{T}$. The reaction rate is expressed as

$$
r^{\text {phase }}=k_{f}^{\text {phase }} \mathfrak{R}^{\text {phase }}\left(\mathbf{x}^{\text {phase }}\right)
$$

with $k_{f}$ as the rate constant of the forward reaction at boiling temperature and $\mathfrak{R}$ as the normalized reaction rate. Accounting for a possible liquid phase splitting, the overall molar liquid holdup $H_{L}$, the mole fraction $x_{i}$ and the catalyst holdups are:

$$
\begin{aligned}
& H_{L}=H_{L}^{\prime}+H_{L}^{\prime \prime}, \\
& x_{i}=\beta x_{i}^{\prime}+(1-\beta) x_{i}^{\prime \prime}, \\
& H_{c a t}^{\prime}=\beta H_{c a t} \text { and } H_{c a t}^{\prime \prime}=(1-\beta) H_{c a t},
\end{aligned}
$$

where $\beta$ is denoted as the relative holdup of one liquid phase with $\beta=H_{L}^{\prime} / H_{L}$.

\section{Reactive condenser}

For the reactive condenser, the component and total mass balances are given by:

$$
\begin{aligned}
& \frac{d\left(H_{V} y_{i}\right)}{d t}+\frac{d\left(H_{L} x_{i}\right)}{d t}=-L x_{i}+v_{i}\left(H_{c a t, c}^{\prime} r^{\prime}+H_{c a t, c}^{\prime \prime} r^{\prime \prime}\right) \quad i=1, \ldots, N C, \\
& \frac{d H_{V}}{d t}+\frac{d H_{L}}{d t}=-L+v_{T}\left(H_{c a t, c}^{\prime} r^{\prime}+H_{c a t, c}^{\prime \prime} r^{\prime \prime}\right),
\end{aligned}
$$

where $L$ stands for the flow rate of the condensate which is withdrawn from the vessel (see Figure 1a). Assuming that the molar liquid holdup in the condenser is much smaller than the vapor holdup, $H_{L} \ll H_{V}$, expanding the derivative in Eqs. 6 and incorporating Eq. 7 yield

$$
H_{V} \frac{d y_{i}}{d t}=-L\left(x_{i}-y_{i}\right)+\left(v_{i}-v_{T} y_{i}\right)\left(H_{c a t, c}^{\prime} r^{\prime}+H_{c a t, c}^{\prime \prime} r^{\prime \prime}\right) \quad i=1, \ldots, N C
$$

Dividing Eqs. 8 by $L$ gives, after little rearrangement, the set of dimensionless balances:

$$
\frac{d y_{i}}{d \xi}=-\left(x_{i}-y_{i}\right)+\left(v_{i}-v_{T} y_{i}\right) \frac{L_{0}}{L} D a_{c} \Theta \quad i=1, \ldots, N C
$$


with $d \xi=\left(L / H_{V}\right) d t$, the condenser Damköhler number $D a_{c}=H_{c a t, c} k_{f, r e f} / L_{0}$ with $k_{f, r e f}$ as rate constant at fixed reference conditions, and the overall reaction term $\Theta$

$$
\Theta=\frac{k_{f}^{\prime}}{k_{f, r e f}} \beta \Re^{\prime}\left(\mathbf{x}^{\prime}\right)+\frac{k_{f}^{\prime \prime}}{k_{f, r e f}}(1-\beta) \Re^{\prime \prime}\left(\mathbf{x}^{\prime \prime}\right),
$$

which represents the overall reaction driving force in the liquid phases. In Eq. $9 \mathrm{~b}, k_{f}^{\prime}$ and $k_{f}^{\prime}$, refer to the corresponding liquid phases. Applying the cooling policy $L=L_{0}=$ constant allows to rewrite Eq. 9a as follows:

$$
\frac{d y_{i}}{d \xi}=-\left(x_{i}-y_{i}\right)+\left(v_{i}-v_{T} y_{i}\right) D a_{c} \Theta \quad i=1, \ldots, N C
$$

In Eqs. 10, the vapor phase mole fractions $y_{i}$ are the dynamic variables while the liquid phase mole fractions $x_{i}$ are algebraic variables. Note that $y_{i}$ and $x_{i}$ have to fulfill the vapor-liquidliquid equilibrium conditions (VLLE) in case of heterogeneous liquid mixtures, and the vapor-liquid equilibrium conditions (VLE) in homogeneous mixtures. For the latter case, the reaction term $\Theta$ in Eqs. 10 simplifies to

$$
\Theta=\frac{k_{f}}{k_{f, r e f}} \mathfrak{R}(\mathbf{x}) .
$$

\section{Reactive reboiler}

For the reactive reboiler, the component and overall mass balances are given by:

$$
\begin{aligned}
& \frac{d\left(H_{V} y_{i}\right)}{d t}+\frac{d\left(H_{L} x_{i}\right)}{d t}=-V y_{i}+v_{i}\left(H_{c a t, r}^{\prime} r^{\prime}+H_{c a t, r}^{\prime \prime} r^{\prime \prime}\right) \quad i=1, \ldots, N C, \\
& \frac{d H_{V}}{d t}+\frac{d H_{L}}{d t}=-V y_{i}+v_{T}\left(H_{c a t, r}^{\prime} r^{\prime}+H_{c a t, r}^{\prime \prime} r^{\prime \prime}\right),
\end{aligned}
$$

where $V$ stands for the flow rate of the vapor which is withdrawn from the vessel (see Figure 1b). If the molar vapor holdup in the reboiler is much smaller than the liquid holdup, $H_{V}<<$ $H_{L}$, with the expansion of the derivative in Eq. 12 and incorporation of Eq. 13, the following dimensionless component mass balances are obtained: 


$$
\frac{d x_{i}}{d \zeta}=\left(x_{i}-y_{i}\right)+\left(v_{i}-v_{T} x_{i}\right) \frac{V_{0}}{V} D a_{r} \Theta \quad i=1, \ldots, N C
$$

where $d \zeta=\left(V / H_{L}\right) d t$ and the reboiler Damköhler number $D a_{r}=H_{c a t, r} k_{f, r e f} / V_{0}$. Applying the heating policy $V=V_{0}=$ constant one obtains:

$$
\frac{d x_{i}}{d \zeta}=\left(x_{i}-y_{i}\right)+\left(v_{i}-v_{T} x_{i}\right) D a_{r} \Theta \quad i=1, \ldots, N C .
$$

In Eqs. 15, the $x_{i}$ are the dynamic variables while $y_{i}$ are algebraic variables which are obtained from the VLE or the VLLE conditions.

As discussed by Venimadhavan et al. (1994), the heating policy plays a significant role for the effect of reaction kinetics on the singular points. In this work, the heating policy for the reboiler, $V=V_{0}=$ constant, and the cooling policy for the condenser, $L=L_{0}=$ constant, are chosen to obtain autonomous systems. These policies are also attractive because they are normally adopted in continuous distillation. It is worth noting that Eqs. 10 and 15 can be seen as the differential analogies of the difference equations derived by Chadda et al. (2001) from their flash cascades.

\section{Conditions for Singular Points}

\section{Potential singular point surface}

The right-hand sides of Eqs. 10 and 15 consist each of two terms. The first one is the separation vector representing the effect of distillation. The second one stands for a vector characterizing the mass conversion in the liquid phase(s) due to the chemical reaction. For non-reactive systems only the separation vector plays a role. For kinetically controlled reactive systems, both vectors may dominate the behavior depending on the value of the Damköhler number. For $D a \rightarrow \infty$, the liquid mixture approaches its chemical equilibrium composition. As condition for a singular point, the change in concentrations due to distillation is exactly balanced by the change in concentrations due to the chemical reaction. 
For the reactive condenser, at a singular point the vapor phase composition $y_{i}$ does not change in time, i.e. $\left(d y_{i} / d \xi\right)=0$. In analogous manner, for the reactive reboiler $\left(d x_{i} / d \zeta\right)=0$ at a singular point. Thus, the following singularity conditions are obtained from Eqs.10 and 15:

$$
\begin{array}{ll}
\text { Condenser: } 0=-\left(x_{i}-y_{i}\right)+\left(v_{i}-v_{T} y_{i}\right) D a_{c} \Theta & i=1, \ldots, N C-1, \\
\text { Reboiler: } 0=\left(x_{i}-y_{i}\right)+\left(v_{i}-v_{T} x_{i}\right) D a_{r} \Theta & i=1, \ldots, N C-1,
\end{array}
$$

where $D a_{c, r} \in(0, \infty)$ for the kinetically controlled system and $D a_{c, r}=0$ for the non-reactive case. The NCth component mole fraction is calculated from the summation of mole fractions to unity. Applying Eq. 16 to an arbitrary reference component $k$ and eliminating the common term $D a_{c} \Theta$ leads to:

$$
\frac{x_{i}-y_{i}}{v_{i}-v_{T} y_{i}}=\frac{x_{k}-y_{k}}{v_{k}-v_{T} y_{k}} \quad i=1, \mathrm{~L}, N C-2
$$

Rearranging Eq. 18, one gets the rate-independent condition for the singular points in the reactive condenser:

$$
X_{i}=Y_{i} \quad i=1, \mathrm{~L}, N C-2
$$

with $X_{i}$ and $Y_{i}$ as the transformed liquid and vapor mole fractions:

$$
X_{i} \equiv \frac{v_{k} x_{i}-v_{i} x_{k}}{v_{k}-v_{T} x_{k}} \quad \text { and } \quad Y_{i} \equiv \frac{v_{k} y_{i}-v_{i} y_{k}}{v_{k}-v_{T} y_{k}} \quad i=1, \mathrm{~L}, N C-2
$$

Starting from Eqs. 17, it is easy to certify that Eqs. 19 are also valid for the singular points of the reactive reboiler.

Eqs. 19 are the general rate-independent conditions for all singular points of the reactive condenser and the reactive reboiler in homogeneous as well as in heterogeneous systems. This is also true for multi-reaction systems with definitions of $X_{i}$ and $Y_{i}$ proposed by Ung and Doherty (1995a). Generally, in a (NC-1) dimensional composition space with $N R$ chemical reactions, $(N C-N R-1)$ rate-independent conditions, $X_{i}-Y_{i}=0$, fix the Potential Singular Point Surface (PSPS) where all kinds of singular points are located on. For a single-reaction system 
the PSPS is a one-dimensional surface, i.e. a curve. In case of $N R=N C$-1, the whole $(N C-1)$ dimensional composition space is the potential surface for the singular points since there can be a maximum of $(N C-1)$ independent reactions from $N C$ nontrivial species (Aris, 1999).

\section{Reaction kinetic surface}

To fix the singular points, additional $N R$ conditions (i.e. Eq. 16 for the reactive condenser and Eq. 17 for the reactive reboiler) are needed. They can be simplified for different reaction conditions as given in Table 1 . Each condition is represented by a $(N C-1)$ dimensional ratedependent hypersurface. It will be the chemical equilibrium surface at $D a \rightarrow \infty$. Singular points are common points (intersection points or tangential points) of the PSPS with this reaction kinetic surface. Possible singular points are pure components, non-reactive azeotropes $(D a=0)$, kinetic azeotropes $(0<D a<\infty)$, and reactive azeotropes $(D a \rightarrow \infty)$.

One should note that, some solutions are outside the valid composition space without any physical significance. One special solution is the pole $\pi$ of stoichiometric lines when $v_{T} \neq 0$. The location of this pole can be determined from $v_{i}-v_{T} x_{i}=0$ (or $\left.v_{i}-v_{T} y_{i}=0\right)$. For a given reaction, it is always located outside the valid composition space since there should be at least one $v_{i}$ with a sign opposite to $v_{T}$. For the special case $v_{T}=0$, the stoichiometric lines become parallel and no pole $\pi$ exists.

The stability of the singular points is determined from the eigenvalues of the Jacobian matrix. For predicting the feasible products of a continuous reactive distillation column, the stable nodes of the reactive condenser and the reactive reboiler have to be determined. These nodes can be collected in a feasibility diagram, which is very helpful for process design.

In the following, first we will analyze the PSPS of some hypothetical and real reaction systems. In the case of MTBE-synthesis, we will demonstrate how to fix singular points by 
intersecting the PSPS and the kinetic surfaces. Then, we illustrate feasible splits of one-feed reactive distillation column taking the synthesis of isopropyl acetate as example.

It should be mentioned that, in some figures, also regions of negative mole fractions are included for a better understanding of the system features.

\section{Examples for PSPS and Singular Points}

\section{Hypothetical ternary systems}

In the considered hypothetical ternary systems, one chemical reaction will take place

$$
\mathrm{A}+\mathrm{B} \Leftrightarrow \mathrm{C} \text {. }
$$

For this system, there is one rate-independent condition for singular points. Using $\mathrm{C}$ as the reference component and arbitrarily choosing $\mathrm{A}$ as the independent variable to represent the system, the equation for the PSPS is given by:

$$
X_{A}=Y_{A}
$$

with the transformed variables

$$
X_{A}=\frac{x_{A}+x_{C}}{1+x_{C}} \text { and } Y_{A}=\frac{y_{A}+y_{C}}{1+y_{C}} .
$$

First let us consider ideal liquid mixture with constant relative volatilities $\alpha_{i j}$. The VLE is described as:

$$
y_{i}=\frac{\alpha_{i k} x_{i}}{\sum_{j=1}^{N C} \alpha_{j k} x_{j}}
$$

Two volatility sequences are considered:

Example 1: $\alpha_{\mathrm{AC}}=0.2, \alpha_{\mathrm{BC}}=3$, i.e. the reaction product $\mathrm{C}$ is the intermediate boiler.

Example 2: $\alpha_{\mathrm{AC}}=5, \alpha_{\mathrm{BC}}=3$, i.e. the reaction product is highest boiler.

Consequently Eq. 22 is a quadratic form in terms of the liquid mole fractions $x_{A}$ and $x_{B}$ : 


$$
\frac{\left(x_{A}-1 / 2\right)^{2}}{\alpha_{B C}-1}-\frac{\left(x_{B}-1 / 2\right)^{2}}{\alpha_{A C}-1}=\frac{\alpha_{A C}-\alpha_{B C}}{4\left(\alpha_{A C}-1\right)\left(\alpha_{B C}-1\right)} .
$$

Depending on the values of the relative volatilities $\alpha_{\mathrm{AC}}$ and $\alpha_{\mathrm{BC}}$ the PSPS shape is fixed:

$$
\left(\alpha_{B C}-1\right) \cdot\left(\alpha_{A C}-1\right) \begin{cases}<0 & \text { ellipse } \\ =0 & \text { parabola } \\ >0 & \text { hyperbola }\end{cases}
$$

The ellipse and hyperbola types are shown in Figure $2 \mathrm{a}$ and $2 \mathrm{~b}$, respectively. The PSPS go through all pure component vertices and the stoichiometric pole $\pi$ of $\boldsymbol{x}=(1,1)$ since $v_{T}=-1$. For the ellipse type (example 1; Figure 2a), the PSPS does not move into the triangle. It intersects with the chemical equilibrium surface only at the pure components A and B. Consequently there exists no reactive azeotrope, and all azeotropes are located outside the triangle. A reactive azeotrope $(\mathrm{RA}=\mathrm{a}$ non-pure component intersection of the PSPS with the chemical equilibrium surface) is located, if $\mathrm{C}$ is the highest boiler (example 2; Figure $2 \mathrm{~b}$ ). This is in agreement with the general conclusion by Barbosa and Doherty (1988) that reactive azeotropes can exist only if the volatilities of the reactants are either all higher or all lower than the volatilities of the products.

If the liquid mixture is extremely non-ideal, liquid phase splitting will occur. Here, the physical properties are adopted from Ung and Doherty (1995a) and Qi et al. (2002) for this hypothetical ternary system. The catalyst is assumed to be equally distributed among the two liquid phases. The corresponding PSPS is depicted in Figure $2 \mathrm{c}$ together with the liquid-liquid envelope and the chemical equilibrium surface. The PSPS passes through the vertices of pure A, B, C, and the pole $\pi$. The shape of the PSPS is affected significantly by the liquid phase non-idealities. As a result, there are three binary non-reactive azeotropes located on the triangle edges and one ternary heterogeneous non-reactive azeotrope inside the triangle. As most interesting feature, one branch of the PSPS is an ellipse-shaped isola moving through the A-vertex and the non-reactive azeotropes ( $\mathrm{AB}, \mathrm{AC}$ and $\mathrm{ABC}$-azeotropes). This PSPS branch 
also locates a ternary heterogeneous reactive azeotrope with the chemical equilibrium surface inside the liquid-liquid region. Between components B and C, the PSPS is evolved from the hyperbola-type PSPS of ideal system depicted in Figure $2 b$.

\section{Real ternary system: MTBE-synthesis}

MTBE is produced by reacting isobutene and methanol $(\mathrm{MeOH})$ in the liquid phase:

$$
\text { Isobutene }+\mathrm{MeOH} \Leftrightarrow \mathrm{MTBE} \text {. }
$$

Venimadhavan et al. $(1994,1999)$ studied the effect of the reaction kinetics on the singular point bifurcations of this system in a reactive reboiler. These authors reported one kinetic pinch point at $D a_{r}=0.166$. Below we will describe the PSPS and locate the singular points by intersecting the PSPS with the kinetic surfaces. The VLE parameters and the kinetics are taken from Venimadhavan et al. (1994). As in their work, the system pressure is $8.11 \times 10^{5}$ $\mathrm{Pa}$.

For this system, we take MTBE as the reference component and choose $\mathrm{MeOH}$ as the independent variable to represent the system. The PSPS is described by

$$
X_{\mathrm{MeOH}}=Y_{\mathrm{MeOH}}
$$

with the transformed variables

$$
X_{M e O H}=\frac{x_{M e O H}+x_{M T B E}}{1+x_{M T B E}} \text { and } Y_{M e O H}=\frac{y_{M e O H}+y_{M T B E}}{1+y_{M T B E}}
$$

Figure 3 predicts the PSPS and the chemical equilibrium surface. The PSPS has a hyperbolatype shape and passes through all pure component vertices and the stoichiometric pole $\pi$. It intersects the isobutene- $\mathrm{MeOH}$ edge and the $\mathrm{MeOH}-\mathrm{MTBE}$ edge at two points, which are non-reactive binary azeotropes. From Figure 3, one can see that there exists no reactive azeotrope in this system. All the bifurcation branches and the pure component vertices, as given in Figure 6 of Venimadhavan et al. (1999), are located on the PSPS. 
In Figure 4, the PSPS is shown together with the kinetic surfaces at four selected reboiler Damköhler numbers. At nonreactive conditions, $D a_{r}=0$ (Figure 4a), one part of the kinetic surface is the line $x_{\mathrm{MeOH}}=0$ which intersects the PSPS at the pure isobutene vertex (saddle point, o ) and the pure MTBE vertex (stable node, I ). Another part of the kinetic surface intersects with the PSPS at the isobutene- $\mathrm{MeOH}$ azeotrope (unstable node, ${ }^{\circ}$ ), the $\mathrm{MeOH}-$ MTBE azeotrope (saddle point) and the pure $\mathrm{MeOH}$ vertex (stable node).

At increasing $D a_{r}$-numbers, the isobutene-MeOH azeotrope moves out of the composition triangle, while the MeOH-MTBE azeotrope moves into the triangle (Figure 4b). The latter point meets the stable node coming from the MTBE-vertex at a Damköhler number of $D a_{r}=$ 0.166. At this $D a_{r}$, the PSPS and the kinetic surface have a common tangent point (kinetic tangent pinch) as shown in Figure 4c. For $D a_{r}>0.166$ (Figure 4d), only three singular points remain in the system: pure $\mathrm{MeOH}$ which is a stable node at any Damköhler number, pure isobutene which is a saddle point at any Damköhler number, and the above mentioned unstable node which is located outside the triangle.

As one can see from Figure 4, the kinetic tangent pinch point at the critical Damköhler number $D a_{r}=0.166$ has an important role for the topology of the maps. This is also reflected by the feasibility diagrams given in Figure 5a,b,c. In Figure 5c, the stable node branch at positive Damköhler numbers are collected from the singular point analyses of the reactive condenser (Figure 5a) and the reactive reboiler (Figure 5b). At Damköhler numbers $D a_{c}>$ 0.085 and $D a_{r}>0.166$, pure isobutene and pure $\mathrm{MeOH}$ are feasible top and bottom products, respectively. At $D a_{r}<0.166$, both pure $\mathrm{MeOH}$ and a kinetic azeotrope (i.e. the mixture on the branch from MTBE to the pinch point) are possible bottom products while another kinetic azeotrope (i.e. the mixture on the branch between isobutene and the non-reactive azeotrope isobutene- $\mathrm{MeOH}$ ) is the possible top product. 


\section{Real quarternary reaction system: Isopropyl Acetate synthesis}

The esterification of acetic acid (HOAc) with isopropanol (IPA) to form isopropyl acetate (IPOAc) is a reversible reaction:

$$
\mathrm{HOAc}+\mathrm{IPA} \Leftrightarrow \mathrm{IPOAc}+\text { Water. }
$$

For this system, Venimadhavan et al. (1999) have studied the bifurcation of the singular points in a reactive reboiler and Chadda et al. (2001) demonstrated their flash cascade idea. Here, the same thermodynamic properties and kinetic expression are used (Table 3 in Venimadhavan et al., 1999).

Since one has only one reaction in this quarternary system, there are two rate-independent conditions for the singular points. IPOAc is taken as reference component, and HOAc and IPA are used to represent the PSPS:

$$
X_{H O A C}=Y_{H O A C} \text { and } X_{I P A}=Y_{I P A}
$$

with transformed variables

$$
\begin{aligned}
& X_{H O A C}=x_{H O A C}+x_{I P O A C} \text { and } Y_{H O A C}=y_{H O A C}+y_{I P O A C} \\
& X_{I P A}=x_{I P C}+x_{I P O A C} \text { and } Y_{I P A}=y_{I P A}+y_{I P O A c} .
\end{aligned}
$$

The surfaces described by Eqs. 31a and 31b in the 3-dimensional composition space intersect with each other and yield the PSPS as curves (Figure 6a). The PSPS contain several branches. Three of them, which pass through the pure components HOAc, IPOAc and water, are located outside the composition space and are not depicted. The branch passing through the IPAvertex locates four non-reactive azeotropes, i.e. IPA-IPOAc, IPOAc-Water, IPA-IPOAcWater, and IPA-Water. This branch also contains the reactive azeotrope (the chemical equilibrium surface is not depicted in order to avoid confusion in the same figure). The PSPS is also displayed in the transformed composition space (Figure 6b). 
The corresponding feasibility diagrams are given in Figure 7, which collects the stable nodes obtained from the intersection of the PSPS and the kinetic surfaces of the reactive condenser and the reactive reboiler. Note that the diagram in Figure $7 \mathrm{a}$ is given in terms of the transformed mole liquid phase fractions $X_{i}$ because these variables allow a geometric illustration of feasible splits, as outlined in the next section. There are two branches where potential bottom products are located, i.e. the pure HOAc branch at any Damköhler number and the pure IPA branch at $1.22<D a<1$. Only the branch between non-reactive azeotrope IPA-IPOAc-Water and reactive azeotrope contains potential top products. As a consequence, there are possible two feed regions yielding two different bottom products (Figure 7a: region I for pure IPA, region II for pure HOAc).

\section{Feasible Split}

Based on Figure 7a, a feasible split of a single-feed fully reactive distillation column at specified feed composition can be predicted. First, the operating equations are derived in terms of the transformed mole fractions. The assumption of constant molar overflow (CMO) is applied and the general equilibrium stage approach is taken. The stage index $j=1$ indicates the reactive condenser and $j=N T$ the reactive reboiler. For a general stage $j$, the component mass balances is given by:

$$
L_{j-1} x_{i, j-1}+V_{j+1} y_{i, j+1}-L_{j} x_{i, j}-V_{j} y_{i, j}+v_{i} D a R_{j}=0
$$

Applying Eq. 34 to component $k$ and eliminating the common term $D a R_{j}$ yields

$$
\begin{aligned}
L_{j-1}\left\lfloor x_{i, j-1}\right. & \left.\left.\left.-\left(v_{i} / v_{k}\right) x_{k, j-1}\right\rfloor+V_{j+1}\right\rfloor y_{i, j+1}-\left(v_{i} / v_{k}\right) y_{k, j+1}\right\rfloor \\
& -L_{j}\left\lfloor x_{i, j}-\left(v_{i} / v_{k}\right) x_{k, j}\right\rfloor-V_{j}\left\lfloor y_{i, j}-\left(v_{i} / v_{k}\right) y_{k, j}\right\rfloor=0
\end{aligned}
$$

Defining the transformed flow rates and compositions at a general stage $m$ as

$$
\tilde{L}_{m}=L_{m}\left[1-\left(v_{T} / v_{k}\right) x_{k, m}\right], \tilde{V}_{m}=V_{m}\left[1-\left(v_{T} / v_{k}\right) y_{k, m}\right]
$$




$$
\begin{gathered}
X_{i, m}=\frac{x_{i, m}-\left(v_{i} / v_{k}\right) x_{k, m}}{1-\left(v_{T} / v_{k}\right) x_{k, m}}=\frac{v_{k} x_{i, m}-v_{i} x_{k, m}}{v_{k}-v_{T} x_{k, m}}, \\
Y_{i, m}=\frac{y_{i, m}-\left(v_{i} / v_{k}\right) y_{k, m}}{1-\left(v_{T} / v_{k}\right) y_{k, m}}=\frac{v_{k} y_{i, m}-v_{i} y_{k, m}}{v_{k}-v_{T} y_{k, m}},
\end{gathered}
$$

and rewriting Eq. 35 yields

$$
\tilde{L}_{j-1} X_{i, j-1}+\tilde{V}_{j+1} Y_{i, j+1}-\tilde{L}_{j} X_{i, j}-\tilde{V}_{j} Y_{i, j}=0 .
$$

For the reactive condenser and the reactive reboiler the mass balances are

$$
\begin{aligned}
& \tilde{V}_{2} Y_{i, 2}-\tilde{L}_{1} X_{i, D}-\tilde{F}_{D} X_{i, D}=0 \\
& \tilde{L}_{N T-1} X_{i, N T-1}-\tilde{V}_{N T} Y_{i, N T}-\tilde{F}_{B} X_{i, B}=0
\end{aligned}
$$

with $\tilde{F}_{D}=F_{D}\left[1-\left(v_{T} / v_{k}\right) x_{k, D}\right]$ and $\tilde{F}_{B}=F_{B}\left[1-\left(v_{T} / v_{k}\right) x_{k, B}\right]$.

The summation of the mass balances from the condenser up to stage $j$ leads to the operating equation of the rectifying section

$$
\tilde{V}_{j+1} Y_{i, j+1}=\tilde{L}_{j} X_{i, j}+\tilde{F}_{D} X_{i, D} \quad i=1, \mathrm{~L}, N C-2 .
$$

Since $\tilde{V}_{j+1}=\tilde{L}_{j}+\tilde{F}_{D}$, Eq. 40 can be modified to get

$$
Y_{i, j+1}=\frac{\tilde{r}}{1+\tilde{r}} X_{i, j}+\frac{1}{1+\tilde{r}} X_{i, D} \quad i=1, \mathrm{~L}, N C-2
$$

where $\tilde{r}=\tilde{L}_{2} / \tilde{F}_{D}$.

In a similar way, the operating equation for the stripping section is

$$
X_{i, j-1}=\frac{\tilde{s}}{1+\tilde{s}} Y_{i, j}+\frac{1}{1+\tilde{s}} X_{i, B} \quad i=1, \mathrm{~L}, N C-2
$$

where $\tilde{s}=\tilde{V}_{N T} / \tilde{F}_{B}$.

The mass balances for the column are

$$
\begin{aligned}
& \tilde{F}_{F} X_{i, F}=\tilde{F}_{D} X_{i, D}+\tilde{F}_{B} X_{i, B} \quad i=1, \mathrm{~L}, N C-2 \\
& \tilde{F}_{F}=\tilde{F}_{D}+\tilde{F}_{B}
\end{aligned}
$$

with the definition $\tilde{F}_{F}=F_{F}\left[1-\left(v_{T} / v_{k}\right) x_{k, F}\right]$.

From Eqs. 43 and 44 one gets 


$$
\frac{\tilde{F}_{D}}{\widetilde{F}_{B}}=\frac{X_{i, B}-X_{i, F}}{X_{i, F}-X_{i, D}} \quad i=1, \mathrm{~L}, N C-2
$$

which can be rewritten as

$$
\frac{s}{1+r} \frac{\left[1-\left(v_{T} / v_{k}\right) x_{k, D}\right]}{\left[1-\left(v_{T} / v_{k}\right) x_{k, B}\right]}=\frac{X_{i, B}-X_{i, F}}{X_{i, F}-X_{i, D}} \quad i=1, \mathrm{~L}, N C-2
$$

where $F_{D} / F_{B}=s /(1+r)$ according to the CMO assumption.

Eq. 45 can also be used to obtain a direct relationship between the feed, distillate and bottom compositions, i.e.

$$
\frac{X_{1, B}-X_{1, F}}{X_{1, F}-X_{1, D}}=\frac{X_{i, B}-X_{i, F}}{X_{i, F}-X_{i, D}} \quad i=1, \mathrm{~L}, N C-2 .
$$

Since the form of the transformed mass balances and the operating equations for the kinetically controlled reactive distillation are identical to the corresponding equations for conventional countercurrent distillation, we therefore can apply the same procedure for designing a reactive distillation column based on the transformed variables. The main idea is to use the feasibility diagram to determine the reflux and reboil ratios, and the Damköhler number. Moreover, the operating equations can be used to determine the stage numbers and the feed position. The whole procedure consists of the following five steps:

1. For the given system determine the PSPS and the singular points by intersecting the PSPS with the kinetic surfaces for the reactive condenser and the reactive reboiler.

2. Collect the stable nodes along the singular point branches and display them in feasibility diagrams (Fig. 7a: composition space in terms of transformed liquid mole fractions; Fig. 7b: composition with respect to the Damköhler number).

3. For a given feed composition $X_{F}$, specify a desired potential bottom product $X_{B}$ (or top product $X_{D}$ ). Then, find the top product $X_{D}$ (or bottom product $X_{B}$ ) from the overall mass balance that is a straight line, on which $X_{B}, X_{F}$, and $X_{D}$ are located (Eq. 47). According to $X_{D}$ (or $X_{B}$ ) one can determine the corresponding $\mathrm{Da}$. 
4. Select a reflux ratio $r$ and determine the corresponding reboil ratio $s$ from Eq. 46 .

5. Calculate the operating equations for the rectifying section and the stripping section starting from the determined $X_{D}$ and $X_{B}$, until they intersect with each other. During the calculation, the VLE (or VLLE) and the reaction rate will be based on the mole fractions. The molar and transformed variables are transformed to each other. The total stage number and the feed position for the reactive distillation column are fixed. If no intersection can be found, select a new reflux ratio $r$ and repeat steps 4 to 5 . In case that no intersection can be found, a feasible split is not possible for the single-feed reactive distillation column with constant Damköhler number.

Now, the algorithm given above is briefly demonstrated for IPOAc synthesis. Steps 1 and 2 were carried out above and the feasibility diagrams are shown in Figure 7 . For the given $X_{F 1}$ (Figure 8a), pure HOAC is chosen as the bottom. As a result, the top product $\boldsymbol{X}_{D}=(0.425$, 0.656) is determined by extrapolating the straight line between $X_{B}$ and $X_{F 1}$ and by intersecting it the PSPS (One can get the same $X_{D}$ from Eq. 47). From the feasibility diagram (Figure 7b), we can obtain the Damköhler number corresponding to $X_{D}$, i.e. $D a=0.33$. Choosing $r=0.7$ one obtains $s=3.884$ from Eq. 46. Starting from $X_{B}$ and $X_{D}$ we solve the operating equations stage by stage and plot the composition of each stage in Figure 8a until the rectifying and the stripping section intersect. Counting the stage numbers yields the total stage number $(N T=$ 30) and the feed stage $(N f=15)$. To check the correctness of this split, we simulated the reactive distillation column using the equilibrium stage model with the obtained configurations. As can be seen, the simulated profiles are in good agreement with the designed ones (Figure 8a). For detailed comparison, the compositions are listed in Table 2.

Figure $8 \mathrm{~b}$ illustrates the feasible split for the feed composition $X_{F 2}$. Pure HOAc is chosen as bottom product and the corresponding Damköhler number is $D a=5.68$. The predicted split and the simulation results are compared in Figure $8 \mathrm{~b}$ and Table 2. 


\section{Conclusions}

For feasibility studies, fast methods are needed to determine the possible top and bottom products of reactive distillation columns. As has been shown above for several reaction examples, feasible bottom products can be obtained as stable nodes of a reactive reboiler. The top products can be determined as stable nodes of a reactive condenser. All potential singular points of the reboiler and the condenser can be found on a common surface, i.e. the potential singular point surface. Non-idealities of the liquid phase can strongly influence the PSPS and the kinetic surfaces. The locations of singular points are identified geometrically as the intersection points and/or tangential points of the PSPS with the reaction kinetic surface.

The stable nodes of both, the reactive condenser and the reactive reboiler, can be collected in a feasibility diagram for the purpose of column design. Based on the feasibility diagram, a possible design of a single-feed, fully reactive distillation column can be derived. The proposed design method is simple to use and can be geometrically interpreted for ternary and quaternary systems.

As a goal for future work, the proposed design method should be extended to columns with varying Damköhler number along the column coordinate. By changing the local Damköhler number it is possible to circumvent undesired kinetic azeotropes. Due to this degree of freedom, kinetic azeotropes are fundamentally different from non-reactive azeotropes $(D a=0)$ and reactive azeotropes $(D a \rightarrow \infty)$ that are both fixed by thermodynamic properties of the mixture. As a further goal for future research activities, the CMO assumption should be dropped by considering the energy balance. 


\section{Notation}

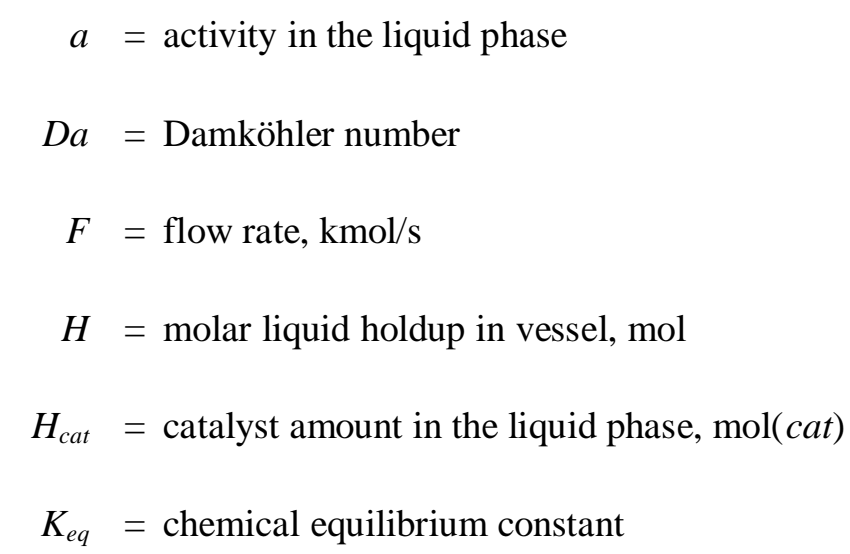

$L, V=$ flow rates of the liquid phase and the vapor phase, respectively, $\mathrm{kmol} / \mathrm{s}$

$N C, N R, N T=$ total numbers of components, chemical reactions and stage numbers, respectively

$\Theta=$ dimensionless reaction term

$r=$ rate of reaction, $\mathrm{mol} / \mathrm{mol}(c a t) \cdot \mathrm{s}$

$r, s=$ reflux ratio and reboil ratio

$R A=$ reactive azeotrope

$\boldsymbol{x}=$ vector of mole fractions in the active liquid phase

$x_{i}=$ overall mole fraction of component $i$ in the liquid phase

$X_{i}, Y_{i}=$ transformed composition in the liquid and vapor phases, respectively

$y_{i}=$ fraction of component $i$ in the vapor phase

Greek letters

$$
\begin{aligned}
\alpha_{i j} & =\text { relative volatility between components } i \text { and } j \\
v_{i} & =\text { stoichiometric coefficient of component } i \\
\xi, \zeta & =\text { dimensionless times } \\
\pi & =\text { pole of stoichiometric lines }
\end{aligned}
$$

\section{Superscripts}

$$
\text { phase }=\text { active liquid phase }
$$




$$
\text { , , ' = extract phase and raffinate phase, respectively }
$$

\section{Subscripts}

$$
0=\text { initial state }
$$

$B, D, F=$ at bottom, distillate and feed of the reactive distillation column

$$
\begin{aligned}
c, r & =\text { reactive condenser and reactive reboiler } \\
k & =\text { reference component } \\
r e f & =\text { at reference temperature } \\
T & =\text { Total }
\end{aligned}
$$

\section{Literature Cited}

Aris R. Mathematical Modelling. London: Academic Press, 1999:154.

Barbosa D, Doherty MF. A new set of composition variables for the representation of reactive-phase diagrams. Proceedings of Royal Society London. 1987;A413:459-464.

Barbosa D, Doherty MF. The influence of equilibrium chemical reactions on vapour-liquid phase diagrams. Chemical Engineering Science. 1988;43:529-540.

Chadda N, Malone MF, Doherty MF. Effect of chemical kinetics on feasible splits for reactive distillation”, AIChE Journal. 2001;47:590-601.

Gadewar SB, Malone MF, Doherty MF. Feasible region for a countercurrent cascade of vapor-liquid CSTRs. AIChE Journal. 2002;48:800-814.

Gadewar SB, Tao L, Malone MF, Doherty MF. Process alternative for reactive distillation. Chemical Engineering Research and Design. 2003; submitted.

Hauan S, Lien KM. Geometric visualizations of reactive fixed points. Computers \& Chemical Engineering. 1996;20:S133-S138.

Hauan S, Westerberg AW, Lien KM. Phenomena-based analysis of fixed points in reactive separation systems. Chemical Engineering Science. 2000;55:1053-1075.

Malone MF, Doherty MF. Reactive distillation. Industrial and Engineering Chemistry Research. 2000; 39:3953-3957. 
Nisoli A, Malone MF, Doherty MF. Attainable regions for reaction with separation. AIChE Journal. 1997;43:374-387.

Qi Z, Kolah A, Sundmacher K. Residue curve maps for reactive distillation systems with liquid phase splitting. Chemical Engineering Science. 2002;57:163-168.

Qi Z, Sundmacher K. Bifurcations study on reactive distillation systems with liquid phase splitting. Computers \& Chemical Engineering. 2002;26:1459-1471.

Rev E. Reactive distillation and kinetic azeotropy. Industrial and Engineering Chemistry Research. $1994 ; 33: 2174-2179$.

Sharma MM, Mahajani SM. Industrial applications of reactive distillation. In: Sundmacher K, Kienle A. Reactive distillation. Weinheim: Wiley-VCH, 2003:3-26.

Song W, Huss RS, Doherty MF, Malone MF. Discovery of a reactive azeotrope. Nature. 1997;388: $561-563$.

Thiel C, Sundmacher K, Hoffmann U. Residue curve maps for heterogeneously catalyzed reactive distillation of fuel ethers MTBE and TAME. Chemical Engineering Science. 1997;52:993-1005.

Ung S, Doherty MF. Vapor-liquid phase equilibrium in systems with multiple chemical reactions. Chemical Engineering Science. 1995a;50:23-48.

Ung S, Doherty MF. Necessary and sufficient conditions for reactive azeotropes in multireaction mixtures. AIChE Journal. 1995b;41:2382-2392.

Venimadhavan G, Buzad G, Doherty MF, Malone MF. Effect of kinetics on residue curve maps for reactive distillation. AIChE Journal. 1994;40:1814-1824.

Venimadhavan G, Doherty MF, Malone MF. Bifurcation study of kinetic effects in reactive distillation. AIChE Journal. 1999;45:546-556. 


\section{Table captions}

Table 1 Necessary condition for singular points in reactive condenser and reactive reboiler (single reaction system).

Table 2 Comparison of mole fractions predicted from the feasible split algorithm and from the simulation (in terms of liquid phase molar fractions $x_{i}$ ).

Table 1

\begin{tabular}{ccc}
\hline & Reactive Condenser & Reactive Reboiler \\
\hline $\begin{array}{c}\text { Non-reactive case } \\
(D a=0)\end{array}$ & $x_{i}-y_{i}=0$ & $x_{i}-y_{i}=0$ \\
Kinetically controlled case & $-\left(x_{i}-y_{i}\right)+\left(v_{i}-v_{T} y_{i}\right) D a_{c} \Theta=0$ & $\left(x_{i}-y_{i}\right)+\left(v_{i}-v_{T} x_{i}\right) D a_{r} \Theta=0$ \\
$(0<D a<\infty)$ & $\left(v_{i}-v_{T} y_{i}\right) \Theta=0$ & \\
Chemical equilibrium & & $\left(v_{i}-v_{T} x_{i}\right) \Theta=0$ \\
controlled case $(D a \rightarrow \infty)$ &
\end{tabular}


Table 2

\begin{tabular}{|c|c|c|c|c|}
\hline & \multicolumn{2}{|c|}{$D a=0.33$} & \multicolumn{2}{|c|}{$D a=5.67$} \\
\hline & $\begin{array}{c}\text { Feasible split } \\
\text { prediction }\end{array}$ & Simulation* & $\begin{array}{c}\text { Feasible split } \\
\text { prediction }\end{array}$ & Simulation $* *$ \\
\hline \multicolumn{5}{|l|}{ Distillate } \\
\hline HOAC & 0.0193 & 0.0193 & 0.0464 & 0.0443 \\
\hline IPA & 0.2498 & 0.2497 & 0.5089 & 0.5065 \\
\hline IPOAc & 0.4057 & 0.4057 & 0.2223 & 0.2247 \\
\hline Water & 0.3252 & 0.3253 & 0.2223 & 0.2245 \\
\hline \multicolumn{5}{|l|}{ Bottom } \\
\hline HOAC & 1.0 & 0.99943 & 1.0 & 0.99956 \\
\hline IPA & 0.0 & 0.00003 & 0.0 & 0.0 \\
\hline IPOAc & 0.0 & 0.00013 & 0.0 & 0.0 \\
\hline Water & 0.0 & 0.00041 & 0.0 & 0.00044 \\
\hline \multicolumn{5}{|c|}{$* \quad N T=30 ; N f=15, r=0.7, s=3.884$} \\
\hline
\end{tabular}




\section{Figure Captions}

Fig. 1 Schemes of batch reactive condenser and batch reactive reboiler.

Fig. 2 Potential singular point surfaces (dash-dot curve) for ternary systems with single reaction $\mathrm{A}+\mathrm{B} \Leftrightarrow \mathrm{C}$. (a) ellipse type, ideal liquid; (b) hyperbola type, ideal liquid; (c) with strongly non-ideal liquid. (RA: reactive azeotrope; solid curve: chemical equilibrium surface).

Fig. 3 Potential singular point surface and chemical equilibrium surface for MTBE reaction system at $8.11 \times 10^{5} \mathrm{~Pa}$.

Fig. 4 Reactive reboiler: Intersections of potential singular point surface with reaction kinetic surface at four different Damköhler numbers $D a_{r}$; MTBE system at $8.11 \times 10^{5} \mathrm{~Pa}$.

Fig. 5 Bifurcation diagrams for reactive condenser (a) and for reactive reboiler (b), and feasibility diagram (c) for MTBE reaction system at $8.11 \times 10^{5} \mathrm{~Pa}$ (dashed curve: chemical equilibrium surface).

Fig. 6 Potential singular point surface for IPOAc reaction system at $1.01 \times 10^{5} \mathrm{~Pa}$. (a) liquid phase composition space in mole fractions $x_{i}$; (b): transformed composition space.

Fig. 7 Feasibility diagrams for IPOAc reaction system at $1.01 \times 10^{5} \mathrm{~Pa}$.

Fig. 8 Design diagram for IPOAc reaction system and comparison with simulation results. (solid curve: simulated column profile; markers: $*=$ stage composition for column rectifying section; + = stage composition for column stripping section). 